Draft VERSion May 20, 2019

Preprint typeset using $\mathrm{LAT}_{\mathrm{E}} \mathrm{X}$ style emulateapj v. 04/20/08

\title{
ON THE ORIGIN OF THE MOLECULAR OUTFLOWS IN IRAS 16293-2422
}

\author{
Josep M. Girart ${ }^{1}$, Robert Estalella ${ }^{2}$, Aina Palau ${ }^{1}$, José M. Torrelles ${ }^{1,2}$, Ramprasad RaO ${ }^{3}$ \\ ${ }^{1}$ Institut de Ciències de l'Espai, (CSIC-IEEC), Campus UAB, Facultat de Ciències, C5p 2, 08193 Bellaterra, Catalonia, Spain, \\ girart@ice.cat \\ 2 Departament d'Astronomia i Meteorologia, Institut de Ciències del Cosmos (UB-IEEC), Martí i Franquès, Universitat de Barcelona, \\ 08028 Barcelona, Catalonia, Spain and \\ ${ }^{3}$ Institute of Astronomy and Astrophysics, Academia Sinica, 645 N. Aohoku Pl., Hilo, HI 96720, USA \\ Draft version May 20, 2019
}

\begin{abstract}
We present $\mathrm{CO} 3-2$, $\mathrm{SiO} 8-7, \mathrm{C}^{34} \mathrm{~S} 7-6$, and $878 \mu \mathrm{m}$ dust continuum subarcsecond angular resolution observations with the Submillimeter Array (SMA) toward the IRAS 16293-2422 (I16293) multiple low-mass protostellar system. The $\mathrm{C}^{34} \mathrm{~S}$ emission traces the $878 \mu \mathrm{m}$ dust continuum well, and in addition clearly shows a smooth velocity gradient along the major axis of component I16293A. CO shows emission at moderate high velocities arising from two bipolar outflows, which appear to be perpendicular with respect to each other. The high sensitivity and higher angular resolution of these observations allows us to pinpoint well the origin of these two outflows at the center of component I16293A. Interestingly, the most compact outflow appears to point toward I16293B. Our data show that the previously reported monopolar blueshifted CO outflow associated with component I16293B seems to be part of the compact outflow arising from component I16293A. In addition, the $\mathrm{SiO}$ emission is also tracing this compact outflow: on one hand, the $\mathrm{SiO}$ emission appears to have a jetlike morphology along the southern redshifted lobe; on the other hand, the $\mathrm{SiO}$ emission associated with the blueshifted northern lobe traces a well defined arc on the border of component I16293B facing I16293A. The blueshifted CO lobe of the compact outflow splits into two lobes around the position of this $\mathrm{SiO}$ arc. All these results lead us to propose that the compact outflow from component I16293A is impacting on the circumstellar gas around component I16293B, possibly being diverged as a consequence of the interaction.
\end{abstract}

Subject headings: ISM: individual objects (IRAS 16293-2422) — ISM: jets and outflows — ISM: molecules — stars: formation

\section{INTRODUCTION}

The dark cloud Lynds $1689 \mathrm{~N}$, located in the Ophiucus star-forming region at a distance of 120 pc (Knude \& Hog 1998; Loinard et al. 2008; Lombardi et al. 2008), harbors IRAS 16293-2422, one of the best studied low-mass (class 0) protostellar systems (hereafter I16293; see Alves et al. 2012; Kristensen et al. 2013; Loinard et al. 2013; Zapata et al. 2013, and references therein). This system has two main components, I16293A and I16293B, separated by $\sim 5^{\prime \prime}(\sim 600 \mathrm{AU})$, first detected at cm-continuum wavelengths (Wootten 1989; Estalella et al. 1991) and later also detected at (sub)mm wavelengths (e.g., Chandler et al. 2005; Rodríguez et al. 2005; Rao et al. 2009; Pineda et al. 2012; Loinard et al. 2013). While I16293A shows an "hourglass" magnetic field structure, I16293B shows an ordered magnetic field (Rao et al. 2009). Wootten (1989) found that I16293A splits into two subcomponents at $\mathrm{cm}$ wavelengths (usually referred as A1 and A2), separated by $0 . \prime 3$ (36 AU) and tracing two stars in a binary system (e.g., Loinard et al.|2007). $\mathrm{H}_{2} \mathrm{O}$ maser emission is also observed toward this binary (Wilking \& Claussen 1987; Wootten 1989; Imai et al. 2007), tracing zones of compressed gas produced by shocks in the presence of very strong line-of-sight magnetic fields ( $\sim 113 \mathrm{mG}$, Alves et al. 2012). I16293 has two bipolar outflows at scales of $\sim 0.1 \mathrm{pc}$ (Walker et al. 1988; Mizuno et al. 1990), one of them centered on I16293A (Yeh et al. 2008). A third, more compact bipo- lar molecular outflow has been also reported through Submillimeter Array (SMA) observations, centered on I16293A and extending along an axis through I16293A and I16293B (SE-NW direction; Rao et al. 2009). The observations indicate that most of the outflow activity in this region is concentrated on the sources within I16293A, with little outflow activity (if any) in the nearby I16293B component, where a compact, possibly isolated protoplanetary disk around a protostar has been inferred (Rodríguez et al. 2005). However, very recent ALMA $\mathrm{CO}$ 6-5 observations with an angular resolution of $\sim 0.3^{\prime \prime}$ led Loinard et al. (2013) to suggest that I16293B is ejecting a blueshifted bubble-like outflow with low velocity and moderate collimation. Based on these results, together with the small kinematic age estimated for this outflow, these authors proposed that I16293B is the youngest object in the region, and one of the youngest protostars known (Loinard et al. 2013).

In this Letter, we present new SMA CO 3-2, $\mathrm{SiO} 8$ 7, $\mathrm{C}^{34} \mathrm{~S} 7-6$, and continuum observations at $345 \mathrm{GHz}$ toward I16293. Our observations are more sensitive to extended structures $\left(\sim 20^{\prime \prime}\right)$ than those of the recently reported $690 \mathrm{GHz}$ ALMA observations (the visibility range for ALMA is 62-943 $\mathrm{k} \lambda$ and for the SMA is 20$240 \mathrm{k} \lambda$ ). Due to these observational properties, our data show that the blueshifted outflow reported previously with ALMA data seems to be part of a bipolar outflow originating from one of the stars within I16293A, rather than originating from I16293B as was recently proposed. 
More importantly, this bipolar outflow is interacting with I16293B producing a shock structure at its SW edge seen in $\mathrm{SiO}$.

\section{OBSERVATIONS}

The SMA observations were taken on 2010 August 28 in the extended configuration. These observations were performed in the polarimetric mode. The results of the polarization data have been presented in a different paper (Rao et al. 2013). The receiver was tuned to cover the $333.5-337.5 \mathrm{GHz}$ and $345.5-349.5 \mathrm{GHz}$ frequencies in the lower side band (LSB) and upper side band, (USB) respectively. The phase center of the telescope was $\mathrm{RA}(\mathrm{J} 2000.0)=16^{\mathrm{h}} 32^{\mathrm{m}} 22 \mathrm{~s} .90$ and $\mathrm{DEC}(\mathrm{J} 2000.0)=$ $-24^{\circ} 28^{\prime} 35^{\prime \prime} 73$. The correlator provided a spectral resolution of about $0.8 \mathrm{MHz}$ (i.e., $0.7 \mathrm{~km} \mathrm{~s}^{-1}$ at $345 \mathrm{GHz}$ ). The gain and bandpass calibrators were QSO J1733130 and QSO 3C454.3, respectively. The absolute flux scale was determined from observations of Neptune. The flux uncertainty was estimated to be $\sim 20 \%$. The data were reduced using the MIRIAD software package (Wright \& Sault 1993). Self-calibration was performed independently for the USB and LSB on the continuum emission of I16293. We have selected the CO 3-2 (345.796 GHz) and $\mathrm{SiO} 8-7$ lines $(347.331 \mathrm{GHz})$, to trace the outflow activity in $\mathrm{I} 16293$, and the $\mathrm{C}^{34} \mathrm{~S}$ 7-6 $(337.396 \mathrm{GHz})$ line to trace the circumstellar gas in the region. In addition, the dust continuum at $878 \mu \mathrm{m}$ is also presented. Maps were obtained from the visibilities using natural weighting, which yielded a synthetic beam size of $\simeq 0^{\prime \prime} 8$, and allowed the tracing of smaller spatial scales $(\simeq 100 \mathrm{AU})$ around I16293 than the scales $(\simeq 300 \mathrm{AU})$ traced by Rao et al. (2009).

\section{RESULTS}

Figure 1 shows the channel maps of the high velocity component of the CO 3-2 emission. This figure shows that the high velocity gas exhibits a quadrupolar morphology well centered in I16293A, apparently forming two bipolar molecular outflows, an extended E-W outflow and a compact NW-SE outflow (the direction of these two outflows are delineated in Fig. 1b). The EW outflow consists of blueshifted emission in the eastern lobe (with some redshifted gas at low flow velocities) and redshifted emission in the western lobe. This bipolar outflow has been previously well studied and extends to distances of $0.1 \mathrm{pc}$ from its powering source (Mizuno et al. 1990; Hirano et al. 2001; Yeh et al. 2008), far beyond the SMA field of view.

The NW-SE bipolar outflow extends only $\simeq 8^{\prime \prime}$ (0.005 pc, $1000 \mathrm{AU})$, so it is very compact compared with the other two bipolar outflows detected in the region (Mizuno et al. 1990). This compact NW-SE bipolar outflow was already reported by Rao et al. (2009), but our new SMA observations now reveal it more clearly (in particular from the $\mathrm{SiO}$ data; see below). At low outflow velocities the blueshifted lobe appears to split in two parts just before reaching I16293B (Fig. 1, and 1 l). At higher velocities (Fig. 1 $a$ and Fig. 1b) the blueshifted gas ends at the position of I16293B. Another characteristic of the outflow is an apparent acceleration of the gas, with the higher velocities appearing farther from the driving source. This is more evident in the position-velocity cut

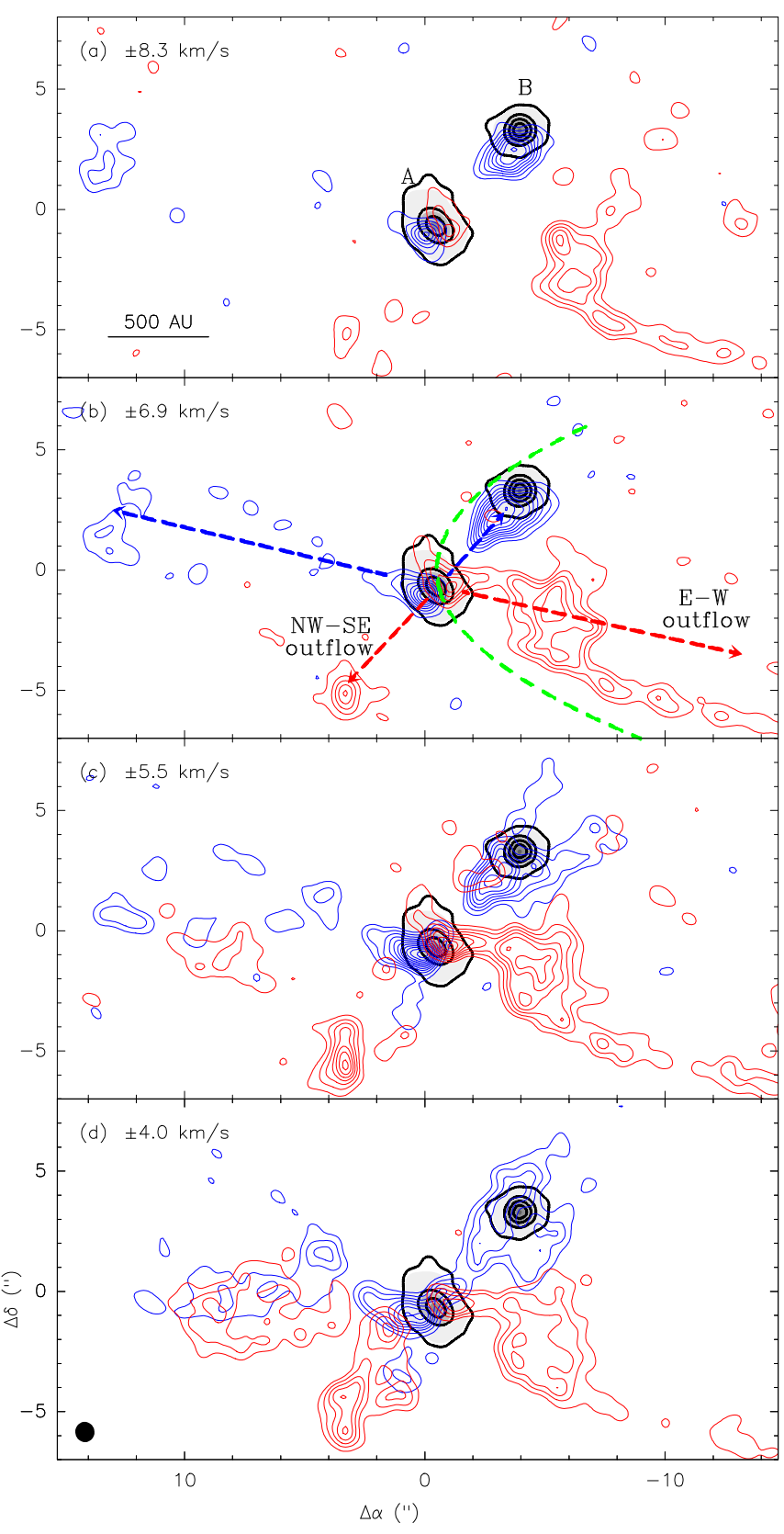

FIG. 1.- Channel maps of the CO 3-2 for the blueshifted (blue contours) and redshifted (red contours) emission, overlapped with the $878 \mu \mathrm{m}$ dust emission (black contours and grey scale). Panels $a, b$ and $c$ : the CO contour's step level and the first contour are $0.47 \mathrm{Jy}$ beam $^{-1}$. Panel $d$ : the CO contour's step level as well the first contour is $0.65 \mathrm{Jy}$ beam $^{-1}$. In all panels the first contour is at 2- $\sigma$ level. The dust contours show the emission at the $5,30,55$, and $80 \%$ level of the maximum intensity, $1.88 \mathrm{Jy}$ beam $^{-1}$. The outflow velocity (i. e., the velocity of the gas with respect to the systemic velocity of $\mathrm{I} 16293 \mathrm{~A}, \simeq 3.5 \mathrm{~km} \mathrm{~s}^{-1}$ ) of each channel is indicated in the top left corner of each panel. The offset spatial positions are with respect to the phase center (given in Section 2). The green dashed line in panel $b$ shows the cavity traced by the E-W CO lobe at scales of $\sim 3000 \mathrm{AU}$ (Yeh et al. [2008). The synthesized beam size of the maps is shown in the bottom left corner of panel $d$.

along the blueshifted lobe (Fig. 2 $2 a$ ): the terminal outflow velocity increases with the distance from the source. A cut across the blueshifted lobe (Fig. 2b) shows that the highest outflow velocities are spatially more compact than the outflow component at velocities closer to the systemic velocity of the I16293A core, suggesting that 


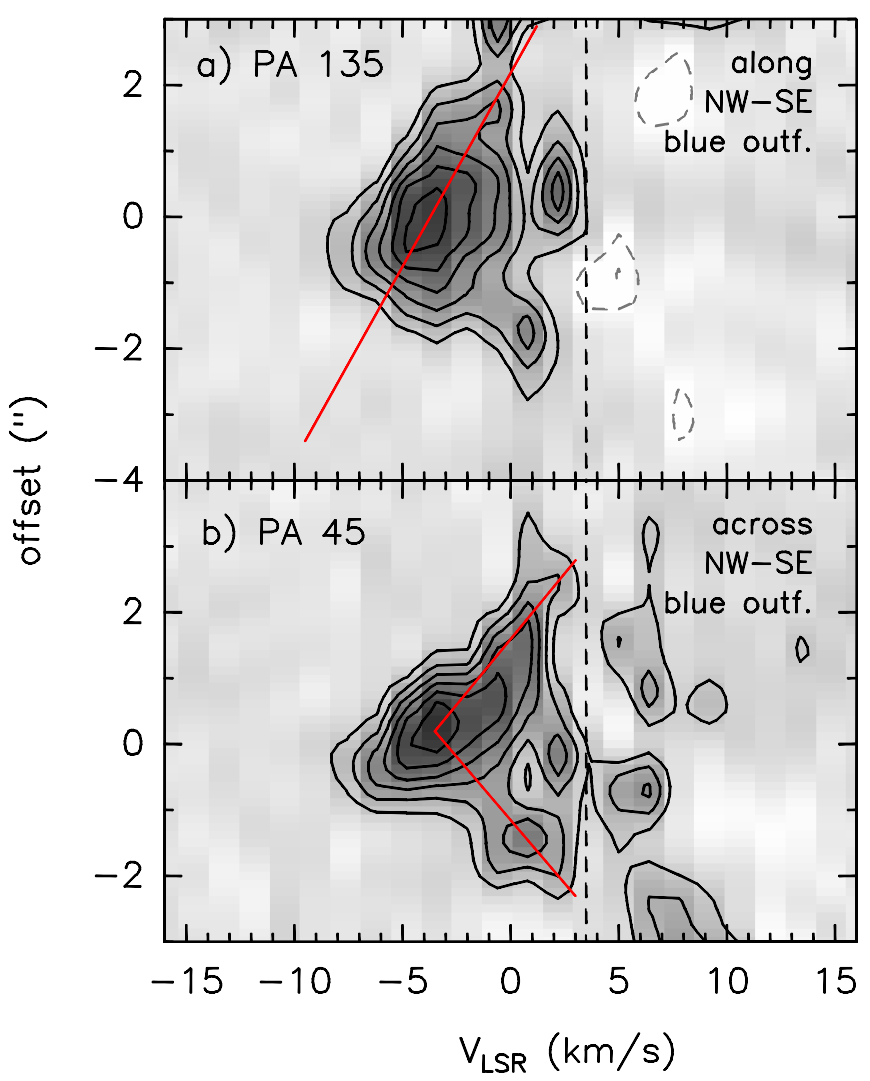

FIG. 2.- Position-velocity plots of the CO 3-2 emission along (panel $a$ ) and across (panel $b$ ) the blueshifted lobe of the NW-SE compact outflow. The reference position ( $0^{\prime \prime}$ offset) is $\mathrm{RA}(\mathrm{J} 2000.0)=16^{\mathrm{h}} 32^{\mathrm{m}} 22^{\mathrm{s}} .65$ and $\mathrm{DEC}(\mathrm{J} 2000.0)=-24^{\circ} 28^{\prime} 33^{\prime \prime} .5$. I16293A, the driving source of this outflow is located approximately at the top of panel $a$ (offset position of $+3^{\prime \prime}$ ). The vertical dashed line indicates the systemic velocity of I16293A. The red lines are shown to better indicate the velocity gradient of the blueshifted $\mathrm{CO}$ emission.

the outflow is more collimated at higher outflow velocities.

The SiO 8-7 emission (Fig. 3) appears to arise mainly from two patches of emission. One of them extends from I16293A, in a jet-like structure, toward the south-east following well the redshifted lobe of the compact NWSE outflow seen in CO 3-2. Most of the emission in the jet-like structure appears close to the cloud velocity but slightly redshifted $\left(v_{\mathrm{LSR}} \simeq 3.0-12.0 \mathrm{~km} \mathrm{~s}^{-1}\right)$. On the other hand, the northern patch of $\mathrm{SiO}$ emission shows a clear partial ring surrounding I16293B, facing I16293A (Fig. 3), and appears near the position where the blue lobe of the CO compact NW-SE outflow diverges spatially (Fig. 1).

The $\mathrm{C}^{34} \mathrm{~S}$ 7-6 mainly traces the circumstellar gas around I16293A at scales of few hundreds AU and matches the $878 \mu \mathrm{m}$ dust emission very well (Fig. (4). Both the circumstellar molecular gas and the dust structures are elongated along the NE-SW direction $(P A \simeq$ $41^{\circ}$, with position velocity cuts of the CS emission along and across the major axis suggesting that the gas is rotating with a Keplerian-like pattern. Below, we describe the procedure to fit the $\mathrm{C}^{34} \mathrm{~S} 7-6$ emission toward I16293A with a rotating geometrically thin disk.

\subsection{Thin Disk Model for I16293A}
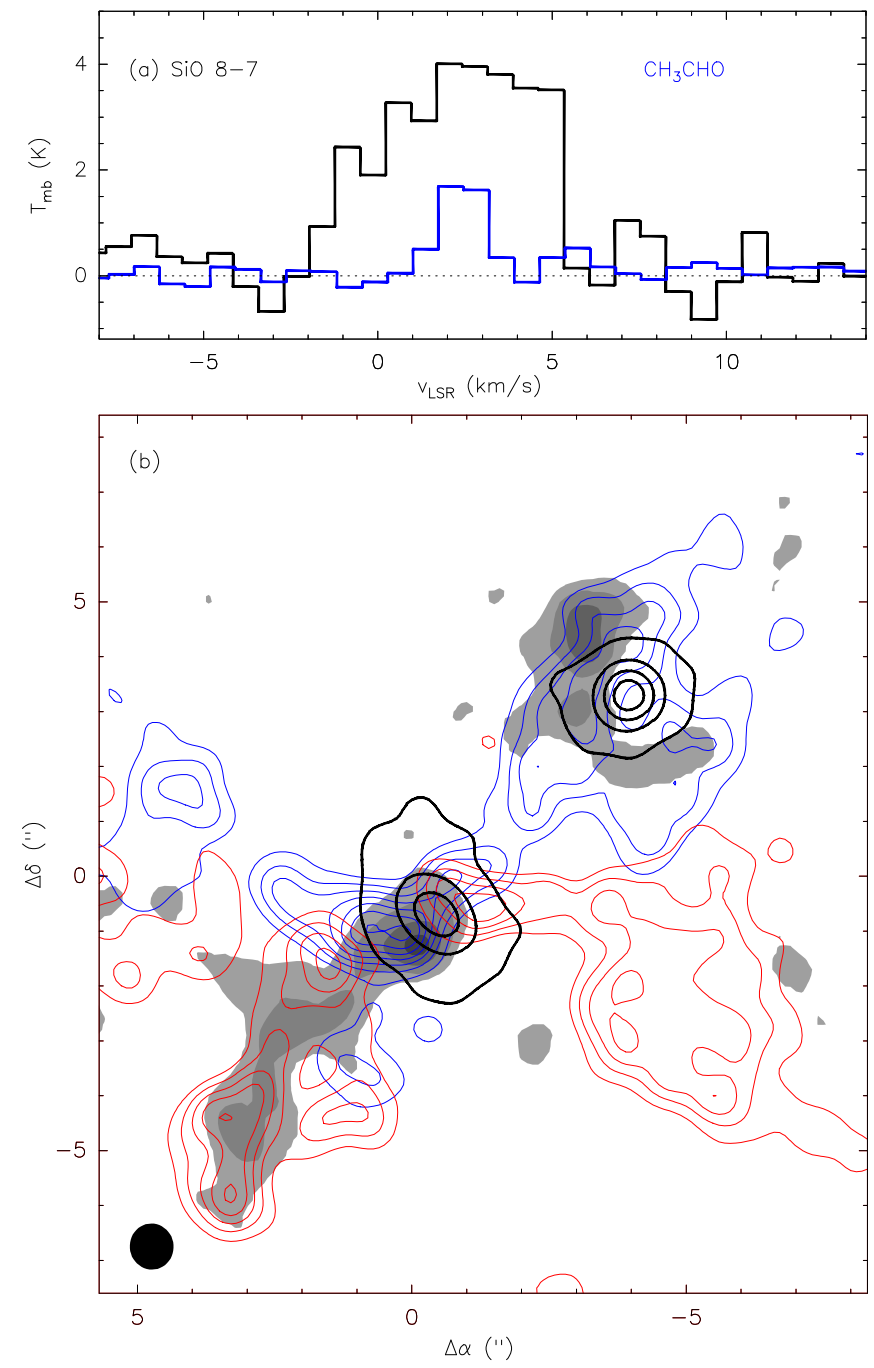

FIG. 3.- Panel a: Spectra of the SiO 8-7 (black spectrum) and $\mathrm{CH}_{3} \mathrm{CHO}$ (blue spectrum) lines, averaged over an area of 9 $\operatorname{arcsec}^{2}$ around I16293B. The $\mathrm{CH}_{3} \mathrm{CHO}$ spectrum was obtained by averaging the $18_{0,18}-17_{0,17} \mathrm{~A}$ and $\mathrm{E}$, and $17_{2,15}-16_{2,14} \mathrm{~A}$ and $\mathrm{E}$ lines (these four lines are located in the 334.9-335.4 GHz range and have similar excitation temperatures and line strengths). Panel $b$ : Zoom in of the panel $d$ from Fig. 1 (contours of the CO and dust emission are the same as those from Fig. 1), overlapped of the integrated $\mathrm{SiO}$ 8-7 emission (grey image).

We considered a geometrically thin disk, with an inner $\left(r_{i}\right)$ and outer $\left(r_{o}\right)$ radius. The angle between the disk axis and the plane of the sky is $i\left(i=0^{\circ}\right.$ for an edge-on disk). We consider a rotation velocity given by a power law of the radius, $v_{r}\left(r / r_{0}\right)^{q_{r}}$, where $r_{0}$ is an arbitrary reference radius and $v_{r}$ is the rotation velocity at the reference radius.

We computed, for each point of a regular grid in the plane of the sky, the projection of the rotation velocity of the corresponding point of the disk along the line of sight $v_{z}$. A Gaussian line profile of width $\Delta v$ and centered on $v_{z}$ was added to the channels associated with the grid point. Finally, each channel map was convolved spatially with a Gaussian beam of width $\Delta s$. However, the intensity scale of the channel maps is arbitrary. A scaling factor, the same for all channel maps, was obtained by minimizing the sum of the squared differences between the data channel maps and the synthetic channel maps. 

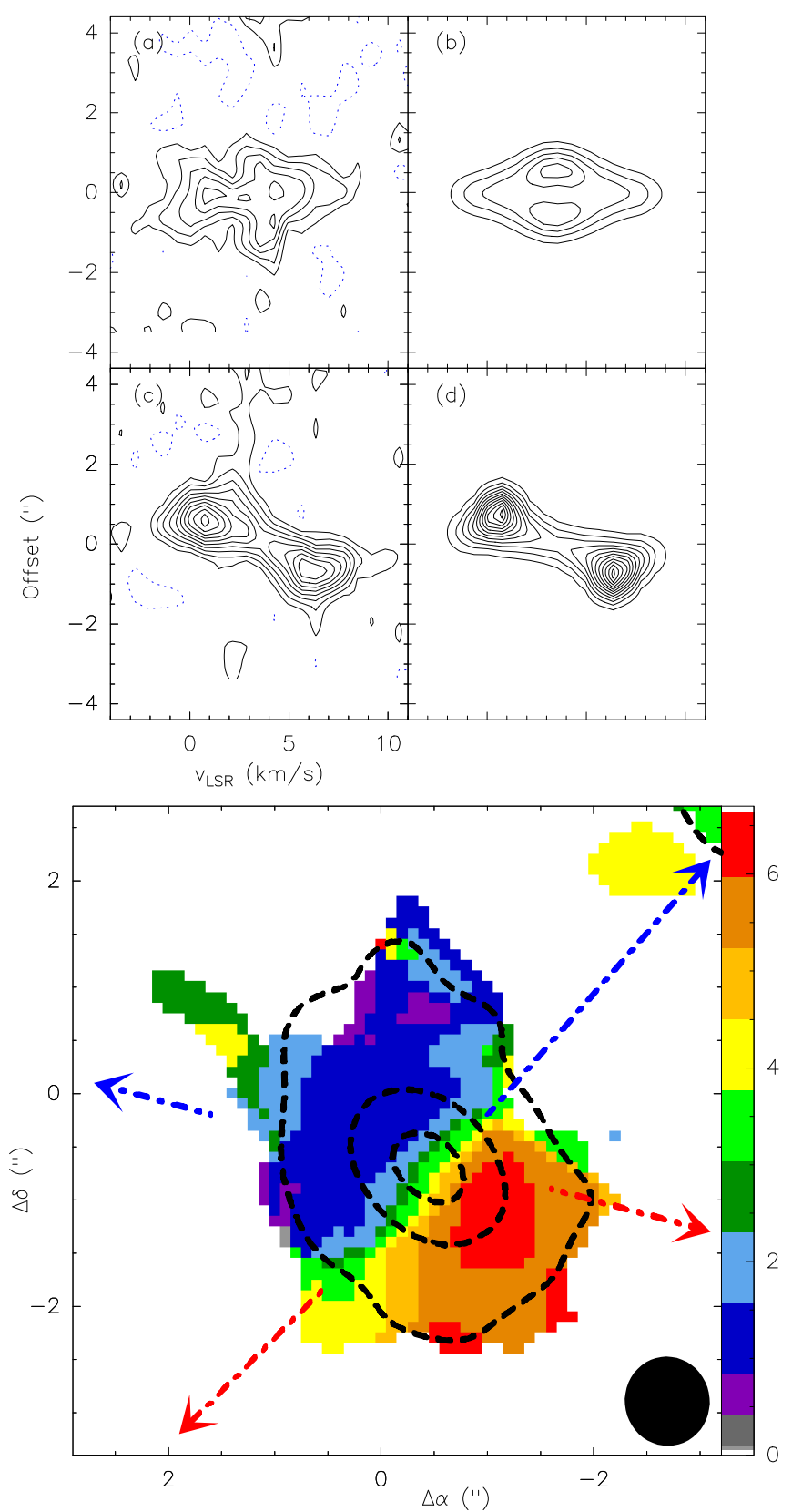

FIG. 4.- Bottom panel: color image of the first order moment (velocity field) of the $\mathrm{C}^{34} \mathrm{~S}$ 7-6 line toward I16293A. The color scale (in $\mathrm{km} \mathrm{s}^{-1}$ ) is shown in the right side of the panel. The black thick dashed contours show the $878 \mu \mathrm{m}$ continuum emission. The thick dashed blue and red arrows show the direction of outflows. Top panels: velocity-position plots of the $\mathrm{C}^{34} \mathrm{~S}$ 7-6 lines taken along the minor (panel $a$ ) and major (panel $c$ ) axes. Panels $b$ and $d$ show the modeled data.

The model depends on a total of 10 parameters, namely the beamwidth, $\Delta s$; the linewidth, $\Delta v$; the disk center, $\left(x_{0}, y_{0}\right)$; the disk systemic velocity, $v_{0}$; the disk inner and outer radii, $r_{i}$ and $r_{o}$; the disk rotation velocity at the reference radius, $v_{r}$; the radial dependence power-law index of the rotation velocity, $q_{r}$; and the disk inclination angle. $i$. Some of the parameters are known beforehand, such as $\Delta s$ and $\Delta v$. Some other can be guessed based on physical grounds (i.e. $q_{r}=-0.5$ ). These considerations leave 7 free parameters, the first 3 are geometrical $\left(x_{0}\right.$, $\left.y_{0}, v_{0}\right)$, and the last 4 have with physical interest $\left(r_{i}, r_{o}\right.$,
TABLE 1

Parameters of the Best Fit Model

\begin{tabular}{lcc}
\hline \hline Parameter & Units & Value \\
\hline FIXED: & $(\operatorname{arcsec})$ & 0.82 \\
Beamwidth $\Delta s$ & $\left(\mathrm{~km} \mathrm{~s}^{-1}\right)$ & 1.20 \\
Linewidth $\Delta v$ & & -0.50 \\
Rotation power-law index $q_{r}$ & & \\
FitTED: & $(\operatorname{arcsec})$ & $-0.12 \pm 0.02$ \\
Disk center $x_{0}$ & $(\operatorname{arcsec})$ & $0.20 \pm 0.01$ \\
Disk center $y_{0}$ & $\left(\mathrm{~km} \mathrm{~s}^{-1}\right)$ & $3.49 \pm 0.09$ \\
Disk systemic velocity $v_{0}$ & $(\mathrm{AU})$ & $1 \pm 1$ \\
Disk inner radius $r_{i}$ & $(\mathrm{AU})$ & $140 \pm 2$ \\
Disk outer radius $r_{o}$ & $\left(\mathrm{~km} \mathrm{~s}^{-1}\right)$ & $-6.5 \pm 0.2$ \\
Rotation velocity $v_{r}{ }^{a}$ & $(\mathrm{deg})$ & $44.2 \pm 0.9$ \\
Disk inclination $i$ &
\end{tabular}

$v_{r}$ and $i$ ), which can be estimated through model fitting to the data.

The fitting procedure was the sampling of the sevendimensional parameter space, using the same procedure as that described in Estalella et al. (2012) and Sánchez-Monge et al. (2013). The parameter space was searched for the minimum value of the rms fit residual. Once a minimum of the rms fit residual was found, the uncertainty in the parameters fitted was found as the increment of each of the parameters of the fit necessary to increase the rms fit residual by a factor of $[1+\Delta(m, \alpha) /(n-m)]^{1 / 2}$, where $n$ is the number of data points fitted, $m$ is the number of parameters fitted, and $\Delta(m, \alpha)$ is the value of $\chi^{2}$ for $m$ degrees of freedom (the number of free parameters) and $\alpha$ is the significance level $(0<\alpha<1)$. For $m=7$, and for a significance level of 0.68 (equivalent to $1 \sigma$ for a Gaussian error distribution), the increment in the rms fit residual is given by $\Delta(7,0.68)=8.17$ (Sánchez-Monge et al. 2013).

The model was fitted to the $\mathrm{C}^{34} \mathrm{~S} 7-6$ emission associated to I16293A. The rotation axis in the plane of the sky (derived from the CS velocity gradient) was found to be at a position angle of -40.8 . The best fit values and their errors are shown in Table 1 . Figure 4 shows the comparison of the synthetic position-velocity cuts for the best solution with those for the SMA C ${ }^{34} \mathrm{~S} 7-6$ data, with the best fit values matching the data very well. The model used assumes that the intensity is proportional to the geometrical depth of the disk along the line of sight (i.e. optically thin emission, and constant density and temperature), which is reasonable for the $\mathrm{C}^{34} \mathrm{~S}$ emission, except near the disk center. Thus, the non-zero value obtained from the fit for the disk inner radius can be only interpreted in the sense that the contribution of the emission near the disk center is low. The simple kinematical model used here cannot discard the idea that the inner radius is actually larger, as suggested by the binarity of the central source, with a semi-major axis of 0.'35 (Loinard et al. 2007).

The mass of the protostar in source A can be estimated from the values derived from the disk fitting. Assuming Keplerian rotation $\left(M=r v^{2} / G\right)$, the mass is $M_{\mathrm{I} 16293 \mathrm{~A}}=2.3 \pm 0.1 M_{\odot}$. This mass is similar to the mass derived from the relative motions of the A1 and A2 objects (Pech et al. 2010, these two objects are embedded in the $\mathrm{C}^{34} \mathrm{~S}$ structure). 


\section{DISCUSSION AND CONCLUSIONS}

The first single-dish observations in I16293 showed two bipolar molecular outflows, one extended along the NE (redshifted)-SW (blueshifted) direction, and another along the $\mathrm{E}$ (blueshifted)-W (redshifted) direction (Walker et al. 1988; Mizuno et al. 1990). The NE-SW outflow has not been detected at smaller scales through interferometric observations, suggesting that it might be a fossil outflow (Yeh et al. 2008; Rao et al. 2009). The E$\mathrm{W}$ outflow has been well detected and studied with the SMA at arcsecond angular resolution (Yeh et al. 2008; Rao et al. 2009). The CO emission associated with the western lobe at scales of $\sim 3000$ AU appears to follow a parabolic cavity with an inclination angle of $30^{\circ}$ with respect to the plane of the sky (see the green dashed line in Figure 1 Yeh et al. 2008). However, our maps show that near the protostars the CO outflow emission does not follow this parabolic cavity. At the position of I16293A the CO 3-2 outflow is very bright and extends roughly in the direction of the E-W outflow. At the highest velocity channel (Fig. 1 1 a) the CO emission around I16293A is compact and the blue and redshifted peaks form a position angle of $\simeq 100^{\circ}$, so they are possibly also associated with the E-W outflow.

The relatively high velocity CO 3-2 emission as traced by the SMA delineates a well defined bipolar compact outflow of only $0.005 \mathrm{pc}$ in the NW-SE direction, well centered around I16293A, as was already suggested by Rao et al. (2009). However, this differs from what has recently been reported from ALMA CO 6-5 observations (Loinard et al. 2013; Kristensen et al. 2013). There are some observational features that support our statement. First, the NW-SE outflow appears to be parallel to the rotation axis of the circumstellar disk-like structure, traced by the $\mathrm{C}^{34} \mathrm{~S}$, around I16293A (see Fig. 4). Second, the overall kinematical and morphological features of the NW blue lobe appear to be very consistent with those of the prototypical molecular outflows associated with class 0 protostars (e. g., Arce \& Sargent 2006; Palau et al. 2006) if it is powered by I16293A: it has a conical-like structure starting in this source; the CO 32 channel maps show an apparent Hubble-like velocity structure (higher velocities arise farther from the powering protostar: see also the position-velocity cut along the blue lobe in Fig. 2); a cut across the blue lobe shows (Fig. 2) that the highest CO 3-2 velocities occur along the outflow axis (i. e., the highest velocities are more collimated than the lowest). Third, the $\mathrm{SiO}$ emission is found only along the SE-NW direction. The $\mathrm{SiO}$ is a molecule that traces shocks strong enough to produce dust sputtering, releasing silicates from the dust mantles (e. g., Anderl et al. 2013). The morphology of the $\mathrm{SiO}$ emission associated with I16293B suggests that the SiO arises from the external shells of the circumstellar material of this component. Its location, facing component I16293A and overlapping with the NW blueshifted lobe, suggests that the SiO traces the region where the NWSE outflow (powered by I16293A) is impacting on the circumstellar gas around I16293B. In fact, the $\mathrm{SiO}$ spectrum of the emission around I16293B is much broader than the acetaldehyde $\left(\mathrm{CH}_{3} \mathrm{CHO}\right)$ spectrum (see Fig 2b). Acetaldehyde is a hot core tracer and is likely tracing the quiescent (apparently unperturbed) circumstellar gas in
I16293B.

The terminal velocity of this compact outflow is small, $\simeq 13 \mathrm{~km} \mathrm{~s}^{-1}$. This outflow is in projection perpendicular to the major axis of the disk-like structure associated with I16293A, so we can fairly assume that this configuration holds in 3-D. Thus correcting for the outflow inclination $\left(44^{\circ}\right.$, see Table 1), we find a dynamical timescale of $\sim 400 \mathrm{yr}$. This is much smaller than the kinematic timescale of the extended E-W outflow (5000 yr, Mizuno et al. 1990). We also estimated the outflow parameters of the compact NW-SE outflow following Palau et al. (2007), and assuming the same inclination given above, optically thin emission, and an excitation temperature of $\sim 18 \mathrm{~K}$ (derived from the line peak of the $\mathrm{CO} 3-2$ spectrum). We obtained a total mass of $\sim 2 \times 10^{-4} M_{\odot}$, a mass outflow rate of $\sim 5 \times 10^{-7} M_{\odot} \mathrm{yr}^{-1}$, and a momentum rate of $\sim 6 \times 10^{-6} M_{\odot} \mathrm{km} \mathrm{s}^{-1} \mathrm{yr}^{-1}$. The momentum rate and the bolometric luminosity of I16293A (somewhat smaller than $\simeq 25 L \odot$, which is the total luminosity in the region) match the correlation between bolometric luminosity and outflow momentum rate found in the literature (Beltrán et al. 2008; Takahashi \& Ho 2012). Yet, the small outflow mass and dynamic time scales suggest that this is a very young molecular outflow, possibly being powered by the youngest protostar in the region. Finally, we note that the water masers detected in the region appear to be redshifted and located only 0.'1 (12 AU) South and South-East $\left(P A=157-182^{\circ}\right)$ from source A1 (Pech et al. 2010; Alves et al. 2012). This suggests that A1 could be the powering source of the compact outflow (its $\mathrm{SE}$ lobe is also redshifted).

In summary, the SMA data presented suggest a scenario where I16293A consists of at least two protostars, one driving the $\mathrm{E}-\mathrm{W}$ outflow, and another driving a more compact and chemically rich NW-SE outflow (possibly A1). Both protostars in I16293A are embedded in a circumbinary disk traced by the $878 \mu \mathrm{m}$ continuum and the $\mathrm{C}^{34} \mathrm{~S}$ 7-6 emission, which is elongated perpendicular to the NW-SE outflow and presents a velocity gradient also perpendicular to this outflow. This situation is similar to what is found in the intermediate-mass protostellar system IRAS $22198+6336$, where a binary is driving two perpendicular outflows, and is embedded in a disk rotating perpendicularly to the most chemically rich outflow (Sánchez-Monge et al. 2010; Palau et al. 2011). Finally, the NW-SE outflow driven by one of the protostars in I16293A seems to be impacting on I16293B, as revealed by the $\mathrm{SiO}$ 8-7 emission showing an arc-morphology at the border of I16293B facing I16293A. The physical and chemical effects of such an interaction on the dynamics and evolution of I16293B remain to be studied.

The SMA, a joint project between the Smithsonian Astrophysical Observatory and the Academia Sinica Institute of Astronomy and Astrophysics, is funded by the Smithsonian Institution and the Academia Sinica. JMG also thanks the SMA staff at Hilo for their support. RE, JMG, AP, and JMT are supported by the Spanish MINECO AYA2011-30228-C03 and Catalan AGAUR 2009SGR1172 grants. The ICC (UB) is a CSICAssociated Unit through the ICE (CSIC). 


\section{REFERENCES}

Alves, F. O., Vlemmings, W. H. T., Girart, J. M., \& Torrelles, J. M. 2012, A\&A, 542, A14

Anderl, S., Guillet, V., Pineau des Forêts, G., \& Flower, D. R. 2013, A\&A, 556, A69

Arce, H. G., \& Sargent, A. I. 2006, ApJ, 646, 1070

Beltrán, M. T., Estalella, R., Girart, J. M., Ho, P. T. P., \& Anglada, G. 2008, A\&A, 481, 93

Chandler, C. J., Brogan, C. L., Shirley, Y. L., \& Loinard, L. 2005, ApJ, 632, 371

Estalella, R., Anglada, G., Rodriguez, L. F., \& Garay, G. 1991, ApJ, 371, 626

Estalella, R., López, R, Anglada, G., Gómez, G., Riera, A., \& Carrasco-González, C. 2012, AJ, 144, 61

Hirano, N., Mikami, H., Umemoto, T., Yamamoto, S., \& Taniguchi, Y. 2001, ApJ, 547, 899

Imai, H., Nakashima, K., Bushimata, T., et al. 2007, PASJ, 59, 1107

Knude, J., \& Hog, E. 1998, A\&A, 338, 897

Kristensen,L. E., Klaassen,P. D., Mottram,J. C., Schmalzl,M., \& Hogerheijde,M. R. 2013, A\&A, 549, L6

Loinard, L., Chandler, C. J., Rodríguez, L. F., et al. 2007, ApJ, 670,1353

Loinard, L., Torres, R. M., Mioduszewski, A. J., \& Rodríguez, L. F. 2008, ApJ, 675, L29

Loinard, L., Zapata, L. A., Rodríguez, L. F., et al. 2013, MNRAS, 430, L10

Lombardi, M., Lada, C. J., \& Alves, J. 2008, A\&A, 480, 785

Mizuno, A., Fukui, Y., Iwata, T., Nozawa, S., \& Takano, T. 1990, ApJ, 356, 184
Palau, A., Estalella, R., Ho, P. T. P., Beuther, H., \& Beltrán, M. T. 2007, A\&A, 474, 911

Palau, A., Fuente, A., Girart, J. M., et al. 2011, ApJ, 743, L32

Palau, A., Ho, P. T. P., Zhang, Q., et al. 2006, ApJ, 636, L137

Pech, G., Loinard, L., Chandler, C. J., et al. 2010, ApJ, 712, 1403

Pineda, J. E., Maury, A. J., Fuller, G. A., et al. 2012, A\&A, 544, L7

Rao, R., Girart, J. M., Marrone, D. P., Lai, S.-P., \& Schnee, S. 2009, ApJ, 707, 921

Rao, R., Girart, J. M., Lai, S.-P., \& Marrone, D. 2013, ApJ, in press, arXiv: 1311.6225

Rodríguez, L. F., Loinard, L., D'Alessio, P., Wilner, D. J., \& Ho, P. T. P. 2005, ApJ, 621, L133

Sánchez-Monge, Á., Palau, A., Estalella, R., et al. 2010, ApJ, 721, L107

Sánchez-Monge, Á., Palau, A., Fontani, F., et al. 2013, MNRAS, 432,3288

Takahashi, S., \& Ho, P. T. P. 2012, ApJ, 745, L10

Walker, C. K., Lada, C. J., Young, E. T., \& Margulis, M. 1988, ApJ, 332, 335

Wilking, B. A., \& Claussen, M. J. 1987, ApJ, 320, L133

Yeh, S. C. C., Hirano, N., Bourke, T. L., et al. 2008, ApJ, 675, 454

Wootten, A. 1989, ApJ, 337, 858

Wright, M. C. H., \& Sault, R. J. 1993, ApJ, 402, 546

Zapata, L. A., Loinard, L., Rodríguez, L. F., et al. 2013, ApJ, 764, L14 